\title{
Papierloses Krankenhaus - gläserner Patient ?
}

Schon vor dem bundesweit publik gewordenen Datenmissbrauch zulasten einer prominenten Patientin des Hamburger Universitätsklinikums (UKE) hatte der Hamburgische Beauftragte für Datenschutz und Informationsfreiheit (HmbBfDI) das Berechtigungskonzept der ehemals staatlichen Krankenhäuser für den Zugriff auf elektronische Patientendaten geprüft. Zusammen mit der nachfolgenden Untersuchung des UKE und einem intensiven Dialog mit den betrieblichen $\mathrm{Da}$ tenschutzbeauftragten der anderen Hamburger Krankenhäuser ergaben sich grundsätzliche Datenschutzprobleme der elektronischen Patientenakte:

Die herkömmliche Vorstellung des Arzt-Patienten-Verhältnisses und auch die Logik der Krankenhausgesetze gehen davon aus, dass nur die Klinikmitarbeiter/ innen, die an der Behandlung eines Patienten konkret beteiligt sind, (möglichst umfassende) Kenntnis der erforderlichen Informationen erhalten: Die klassische Papierakte liegt im Schwesternzimmer der Station zur Einsichtnahme bei konkretem Bedarf und unter den wachsamen Augen des Stationspersonals. Später kontrollieren Archivkräfte die Berechtigung zu Aktenanforderungen.

Die Logik der elektronischen Patientenakte ist eine ganz andere: Mit der stationären Aufnahme des Patienten und seiner Zuweisung zur behandelnden Fachabteilung werden seine Daten automatisch allen Abteilungsmitarbeiterinnen und -mitarbeitern zum Abruf zur Verfügung gestellt. Keine/r muss sich mehr die (Papier-)Akte holen und dies ggf. rechtfertigen; der $\mathrm{Zu}$ griff erfolgt vom Arbeitsplatz aus - schnell, aktuell und vollständig. Was für das tatsächlich behandelnde medizinische und Pflegepersonal - und natürlich für den $\mathrm{Pa}$ tienten - ein Fortschritt ist, wird für die anderen Abteilungsmitarbeiter/innen unter Umständen zur Versuchung: der prominente Filmschauspieler, die attraktive Nachbarin, der nette Kollege von der Urologie, der sich im eigenen Haus behandeln lässt - was fehlt ihnen? Wie ernst sind sie erkrankt? Könnte ich aus der Information
Nutzen ziehen? Die soziale Kontrolle eines Datenzugriffs entfällt weitgehend, möglicherweise auch die Protokollierung des Zugriffs, zumindest ihre systematische Auswertung. Das System sagt, ich darf, jedenfalls kann ich. Das Krankenhausgesetz und der Behandlungsvertrag - wie der Patient ihn versteht - sagen jedoch: ich darf nicht, weil ich nicht konkret an der Behandlung beteiligt bin, oder nur in einem so geringen Maße, dass ich die Patientendaten nicht benötige.

Diese Schere zwischen rechtlichem Dürfen und technischem Können und damit das Risiko eines Datenmissbrauchs kann sich weit öffnen:

- Eine Abteilung mit 20 Ärztinnen und Ärzten und ungleich mehr Pflegepersonen ist in großen Häusern keine Seltenheit; ihre „Zuständigkeit“ für 50 stationäre und täglich weitere 50 ambulante Patienten ebenso wenig. Der einzelne Patient lernt aber nur wenige von ihnen kennen.

- In modernen interdisziplinär ausgerichteten Häusern arbeiten viele Ärzte nicht nur in einer, sondern in mehreren $\mathrm{Ab}$ teilungen, viele Schwestern und Pfleger in mehreren Stationen - mit entsprechenden Zugriffsrechten.

- Wird die Hinzuziehung eines Spezialisten - vom Anästhesisten (Konsil) über den Physiotherapeuten bis zur Sozialarbeiterin - notwendig, erhält zusätzlich auch deren ganze Abteilung, bzw. der zentral eingerichtete Personalpool insgesamt, Zugriff auf die Daten des betroffenen Patienten. Dieses Zugriffsrecht aller Abteilungs- / Pool-Mitarbeiter endet selbst dann nicht, wenn eine/r der Spezialisten die Mitbehandlung übernimmt.

- In aller Regel haben auch die zentralen Funktionsdienste wie das interne Qualitätsmanagement, das Fall-/Patientenmanagement, die Abrechnung, aber auch die Schreibdienste Zugriff auf die Patientendaten.

- Angesichts der technischen Komplexität einer elektronischen Patientenakte verwundert es nicht, dass auch die kli- nikinternen IT-Administratoren, aber in besonderem Maß auch die Spezialisten der Herstellerfirmen Zugriff bis auf die Patientenebene erhalten.

Hinzu kommt: Die in Datenschutz- und Krankenhausgesetzen geforderte Sperrung der Patientendaten nach Entlassung bzw. Behandlungsabschluss scheitert heute weitgehend an den fehlenden technischen Möglichkeiten. Dasselbe gilt für eine Differenzierung der Patientendaten nach Behandlungsfällen: Wer das Zugriffsrecht für einen Patienten hat, hat dies für alle früheren Behandlungsfälle in dem gesamten Krankenhaus - ggf. einschließlich seiner GmbH-Töchter.

Dies soll der Patient in der Regel bereits bei der Aufnahme akzeptieren: Will er behandelt werden, muss er die Klausel des Behandlungsvertrages unterschreiben, dass „die zur Behandlung notwendigen“" Informationen aus früheren Klinikaufenthalten hinzugezogen werden. Dass er damit allen genannten Klinikmitarbeitern die Kenntnisnahme aller früheren Behandlungen ermöglicht, macht sich der Patient kaum klar. Die Wirksamkeit der Einwilligung ist deswegen zweifelhaft.

Bei der Abarbeitung der datenschutzrechtlichen Prüfungen geht es nun darum, die Schere zwischen Dürfen und Können so weit wie medizinisch vertretbar $\mathrm{zu}$ schließen. Dabei sind die Krankenhäuser gefordert, die schon möglichen technischen Optionen zu nutzen. Die Hersteller sind aufgefordert, mehr Datenschutz in ihren Produkten zu implementieren. Für beides hat die Hamburgische Datenschutzaufsicht zusammen mit den betrieblichen Datenschutzbeauftragten der Krankenhäuser einen Katalog von 40 „Normativen Eckpunkten für Zugriffe auf elektronische Patientendaten im Krankenhaus" erarbeitet, den sie nun unter http://www.hamburg.de/datenschutz/ zur Diskussion stellt.

\section{Dr. Hans-Joachim Menzel}

Stellvertretender Hamburgischer Beauftragter für Datenschutz und Informationsfreiheit 